\title{
Hawking Radiation of a Single-Partition Black Hole
}

\author{
Youngsub Yoon $\mathbb{D}$ \\ Department of Physics and Astronomy, Seoul National University, Seoul 08826, Republic of Korea \\ Correspondence should be addressed to Youngsub Yoon; youngsub@post.harvard.edu
}

Received 28 March 2018; Revised 20 May 2018; Accepted 26 June 2018; Published 24 September 2018

Academic Editor: Farook Rahaman

Copyright (C) 2018 Youngsub Yoon. This is an open access article distributed under the Creative Commons Attribution License, which permits unrestricted use, distribution, and reproduction in any medium, provided the original work is properly cited. The publication of this article was funded by SCOAP ${ }^{3}$.

Brian Kong and the present author recently presented a new area spectrum and showed that the new area spectrum implies that the decay time of a single-partition black hole (i.e., a black hole with the area not big enough to have two or more partitions) is roughly constant. In this article, we show why the decay time of a single-partition black hole is roughly constant.

\section{Introduction}

It is now very well-known that black hole emits particles thanks to Hawking's semiclassical treatment of black hole [1]. However, as Rovelli and Smolin discovered loop quantum gravity as a possible method to quantize gravity in late 80 s [2], it was destined that Hawking's semiclassical treatment of Hawking radiation needed some modification. Soon, it was discovered that area is discrete according to loop quantum gravity [3-5], and Rovelli found the first connection between the Bekenstein-Hawking entropy and loop quantum gravity in his seminal paper in 1996 [6]. Soon, Hawking radiation spectrum was also approached from the loop quantum gravity framework using the discreteness of area [7-9]. In mid-2010s, the present author approached the problem of Hawking radiation spectrum from the discreteness of area as well and found surprising results such as full discreteness of Hawking radiation spectrum [10] and Maxwell-Boltzmann nature of Hawking radiation spectrum [11]. Also, Brian Kong and the present author suggested a new area spectrum in [12], boldly claiming that the conventional area spectrum known in loop quantum gravity community could be wrong, if one considers that, for area two-form, one needs to use LeviCivita tensor instead of Levi-Civita symbol as conventionally used in loop quantum gravity community. This consideration predicted that the area spectrum was the square root of the conventional one. As for the evidence of this area spectrum, we showed that the Bekenstein-Hawking entropy was approximately satisfied (we conjectured that it would be exactly satisfied if the effect of extra dimension is considered, which can lead to the prediction of fine structure constant) and found a strange, coincidental numerical relation that implied that the decay time of a single-partition black hole is roughly constant. In this article, we show why the decay time of a single-partition black hole is roughly constant. This new further evidence will give another support the area spectrum proposed by Brian Kong and us. The organization of this paper is as follows. In Section 2, we briefly review loop quantum gravity and its approach to Hawking radiation, particularly focusing on our previous work. In Section 3, we briefly review Brian Kong and the present author's work on new area spectrum focusing on the strange, coincidental numerical relation. In Section 4, we explain our own work on Hawking radiation briefly mentioned in Section 2 in more detail. In Section 5, we explain what is special about singlepartition black hole. In Section 6, we derive the constancy of decay time for single-partition black hole. In Section 7, we conclude our paper.

\section{Loop Quantum Gravity and Its Approach to Hawking Radiation}

According to loop quantum gravity, the eigenvalues of the area operator are quantized [3-5] and the black hole area, as much as any area, is the sum of these eigenvalues. For example, let us say that we have the following area eigenvalues (i.e., the unit areas):

$$
A_{i}=A_{1}, A_{2}, A_{3}, A_{4}, A_{5}, A_{6} \ldots
$$


TABle 1: C(A).

\begin{tabular}{lcccc}
\hline $\mathrm{y}$ & $\mathrm{A}$ & $\mathrm{K}(\mathrm{A})$ & $\mathrm{I}(\mathrm{A})$ & $\mathrm{C}(\mathrm{A})$ \\
\hline 1 & 17.8 & 4 & 767.4 & 191.8 \\
2 & 21.1 & 14 & 2740 & 195.7 \\
3 & 23.4 & 32 & 5552 & 173.5 \\
4 & 25.1 & 50 & 9276 & 185.5 \\
5 & 26.6 & 72 & 14000 & 194.4 \\
6 & 27.8 & 110 & 19814 & 174.1 \\
7 & 28.9 & 154 & 26817 & 172.1 \\
8 & 29.9 & 204 & 35109 & 171.0 \\
9 & 30.8 & 262 & 44797 & 171.7 \\
10 & 31.6 & 326 & 55990 & 177.3 \\
11 & 32.4 & 388 & 68803 & 175.8 \\
12 & 33.1 & 474 & 83353 & 170.8 \\
13 & 33.7 & 584 & 99761 & 172.7 \\
14 & 34.4 & 684 & 118155 & 1386.5 \\
\hline
\end{tabular}

Then, the black hole area $A$ must be given by the following formula:

$$
A=\sum_{i} N_{i} A_{i}
$$

where $N_{i} s$ are nonnegative integers. Here, we can regard the black hole as having $\sum N_{i}$ partitions, each of which has one of the $A_{i}$ as its area.

In [10], the present author showed that the black hole area decrease upon a single emission of photon during Hawking radiation must be given by the unit area. In other words, we have

$$
\Delta A=-A_{i}
$$

As the Bekenstein-Hawking entropy is given by $S=k A / 4$, $[1,13]$ and we know $\Delta Q=T \Delta S$, the energy decrease is given by

$$
\Delta Q=-\frac{A_{i}}{4} k T
$$

Since this energy must be equal to the energy of photon emitted (i.e., $\Delta Q=-E_{\text {photon }}$ ) the energy of the photon emitted during the Hawking radiation is given by

$$
E_{\text {photon }}=\frac{A_{i}}{4} k T
$$

In particular, the Hawking radiation spectrum is discrete.

\section{Area Spectrum and Single-Partition Black Holes}

In [12] (see also [14]), Brian Kong and the present author showed that the area spectrum (i.e., the unit areas) in black hole horizon is given by

A

$$
=8 \pi \sum_{i} \sqrt{\frac{1}{2} \sqrt{2 j_{i}^{u}\left(j_{i}^{u}+1\right)+2 j_{i}^{d}\left(j_{i}^{d}+1\right)-j_{i}^{t}\left(j_{i}^{t}+1\right)}}
$$

with the degeneracy $\left(2 j_{i}^{u}+1\right)+\left(2 j_{i}^{d}+1\right)$ where $j_{i}^{u}, j_{i}^{d}$, and $j_{i}^{t}$ satisfy the triangle inequality (i.e., $j_{i}^{1}+j_{i}^{2} \geq j_{i}^{3}$ ) and $j_{i}^{t}$ is an integer, and both $j_{i}^{u}$ and $j_{i}^{d}$ are integers or halfintegers at the same time. We want to remark that (6) is not a conventional one and it has an extra square root compared to [14]. However, Brian Kong and the present author argued in [12] that we arrive at this formula, if we use, in the equation for the area two-form, a Levi-Civita tensor instead of a Levi-Civita symbol as conventionally done in loop quantum gravity community.

Using this, we calculated the degeneracy of area spectrum using Java. This yielded $K(A)$ in Table 1 where $K(A)$ is the number of states for area equal to or below $A$, and $I(A)$ is given by

$$
I(A)=\int_{A_{\text {cut }}}^{A} \sqrt{A^{\prime}}\left(e^{A^{\prime} / 4}-1\right) d A^{\prime}
$$

and $y$ is conveniently defined as follows:

$$
y=2 j_{i}^{u}\left(j_{i}^{u}+1\right)+2 j_{i}^{d}\left(j_{i}^{d}+1\right)-j_{i}^{t}\left(j_{i}^{t}+1\right)
$$

Of course, using $y$, we have the following relation:

$$
A(y)=8 \pi \sqrt{\frac{1}{2} \sqrt{y}}
$$

$C(A)$ is given by

$$
C(A)=\frac{I(A)}{K(A)}
$$

The point of this table is that $C(A)$ is roughly constant. We found this accidentally. Of course $C(A)$ is not exactly but only approximately constant, but the biggest value for $C(A)$ in our result is 195.7, deviating from the "right value" of $C$, i.e., the value of $C$ for large $A$ by only about 13 percent. As $K(A)$ at this biggest value of $C(A)$ is only 14 , it necessarily has a big "statistical" variation. Therefore, 195.7 is not a big deviation. 
Then, in Sections X and XI of the same paper, we showed that this constancy can be explained if the decay time of a single-partition black hole is constant. Let us briefly summarize some of the main reasonings.

Let us denote the average number of photons emitted from a black hole during the infinitesimal time $\Delta t$ by $j_{\Delta t}$. Then, we have

$$
j_{\Delta t}=\frac{\Delta t}{\tau}
$$

For macroscopic black hole, if $d n_{\text {photon }}$ is the number of photons with a frequency between $v$ and $\nu+d v$ emitted during unit time, from Planck's blackbody radiation formula, we have

$$
d n_{\text {photon }}=\frac{2 \pi v^{2}}{c^{2}} \frac{A_{B H} d v}{e^{h v / k T}-1}
$$

where $A_{B H}$ is the area of black hole. Of course, we naturally have

$$
\frac{1}{\tau}=\int d n_{\text {photon }}
$$

Now let us say that, during the time $\Delta t$, the number of emitted photons which correspond to a decrease in the black hole area by amount $a$ is given by $x_{a(y), \Delta t}$. Here, $y$ is given by (8), and $a(y)$ is given by (9).

Then, in section XI, by some calculations we showed

$$
x_{a, \Delta t}=\frac{3 \sqrt{\pi} c}{2 \sqrt{A_{B H}}} \frac{1}{e^{a / 4}-1} \Delta t
$$

We proceeded by borrowing the idea from a paper by Bekenstein and Mukhanov [7]. The idea is that $x_{a, \Delta t} / j_{\Delta t}$ is proportional to the degeneracy of the black hole after emission divided by the degeneracy of the black hole before emission. Let us denote the degeneracy of the black hole with area $A_{B H}$ by $W\left(A_{B H}\right)$. Then, from (11) and (14), we have

$$
\frac{W\left(A_{B H}-a\right)}{W\left(A_{B H}\right)} \propto \frac{\tau}{\sqrt{A_{B H}}} \frac{1}{e^{a / 4}-1}
$$

Now, if a black hole is a single-partition black hole (a black hole not big enough to have two or more partitions), upon emission of a photon, there is no other value for area decrease than $a=A_{B H}$. Plugging this value to the above formula, we get

$$
\frac{W(0)}{W\left(A_{B H}\right)} \propto \frac{\tau}{\sqrt{A_{B H}}} \frac{1}{e^{A_{B H} / 4}-1}
$$

which implies

$$
W\left(A_{B H}\right) \propto \frac{\sqrt{A_{B H}}}{\tau}\left(e^{\mathrm{A}_{B H} / 4}-1\right)
$$

If $\tau$ is constant for single-partition black hole, then it is precisely our earlier relations (7) and (10). Also, one thing very noticeable is that these relations do not hold for multipartitioned black holes. The minimum area a black hole can have is given by $A(y=1)=4 \pi \sqrt{2}$. For it to be a single-partition black hole, its area should be smaller than $2 A(y=1)=A(y=16)$. Our relations suddenly do not hold beginning from $y=16$ even though it holds very well for $y<16$. This is the reason why the table is presented up to $y=15$.

However, we later realized formula (14), which we derived in section XI of [12], is due to an error. Nevertheless, we obtained a strong hint that Table 1 is due to Hawking radiation of single-partition black hole.

\section{Quantum Corrections to the Hawking Radiation Spectrum}

As mentioned in Section 2, the present author showed in [10] that, upon emission of a single photon during Hawking radiation, only single area quanta can decrease. Let us briefly repeat the main argument in that paper. (In an earlier work [11], the author showed that Hawking radiation should follow Maxwell-Boltzmann statistics rather than Bose-Einstein statistics as considered in [10]. We adjust our presentation here considering this earlier work.)

Consider the following problem [15].

Let us say that the unit areas $A_{1}, A_{2}, A_{3}, \cdots$ have degeneracies $d_{1}, d_{2}, d_{3}, \cdots$. Suppose we have a black hole with area $A$ which satisfies $A=\sum_{i} N_{i} A_{i}$ as explained before in (2). For a given configuration $\left(N_{i}=N_{1}, N_{2}, N_{3}, \cdots\right)$, how many different ways can this be achieved?"

According to Rovelli [6], the area quanta are distinguishable, as they have fixed location on the black hole horizon, and, only if they are so, the Bekenstein-Hawking entropy can be satisfied. Using this distinguishability, the answer to the above question is given by

$$
Q=N ! \prod_{i=1}^{\infty} \frac{d_{i}^{N_{i}}}{N_{i} !}
$$

where $N=\sum_{i} N_{i}$.

By maximizing $Q$ and using (5), one obtains that the Hawking radiation for macroscopic black hole is given by the following Maxwell-Boltzmann distribution:

$$
N_{i}=N d_{i} e^{-h f_{i} /(k T)}=N d_{i} e^{-A_{i} / 4}
$$

(Note that if we do not follow the present author's earlier work [11], this would be the familiar Einstein-Bose distribution of Hawking radiation, namely, $N_{i} / N=d_{i} /\left(\exp \left(h f_{i} / k T\right)-1\right)$.)

We can do better than this. We can express the above expression in terms of the black hole horizon area $A$. By plugging the above formula to (2), we get

$$
N=\frac{A}{\sum_{i} d_{i} A_{i} e^{-A_{i} / 4}}
$$

By plugging the above formula back to (19), we get

$$
N_{i}=\frac{A d_{i} e^{-A_{i} / 4}}{\sum_{i} d_{i} A_{i} e^{-A_{i} / 4}}
$$


Notice that the above expression is proportional to the black hole horizon area $A$. Therefore, we conclude that the Hawking radiation for macroscopic black hole is proportional to the area of black hole, as much as Planck's blackbody radiation spectrum is proportional to the area of blackbody.

\section{Single-Partition Black Hole}

Let us see what happens for (2) for a single-partition black hole. In such a case we have $N=1$. This implies $N_{i}=1$ for the concerned $i$ and 0 for other unconcerned values of $i$. Now, remember what $N_{i}$ meant in (19). It is exactly Hawking radiation spectrum of given frequency. As the sum of $N_{i}$ is $N, N$ is the sum of Hawking radiation of every frequency, namely, total Hawking radiation. Thus, we conclude that the decay time of the single-partition black hole is constant as $N$, which is 1 , is constant. In other words, $\tau$ is constant as desired.

\section{Derivation}

In this section, we derive (7) and (10) using the correct method.

At the end of Section 4, we showed that Hawking radiation is proportional to the black hole area $A$. However, this is only half correct as this holds true only if the temperature of black hole were fixed constant. In reality, the temperature of black hole depends on the black hole area $A$.

So, let us consider this dependence. The number of black hole area quanta is given by (20), and the number of photons emitted per a second is given by (12) and (13). That is,

$$
\frac{1}{\tau}=A_{B H} \frac{2 \pi}{c^{2}} \int_{\pi \sqrt{2}}^{\infty} \frac{u^{2} d u}{e^{u}-1}\left(\frac{k T}{h}\right)^{3}
$$

where the integration range is not from 0 but from $\pi \sqrt{2}$ because the minimum unit area satisfies $A(y=1) / 4=\pi \sqrt{2}$ which is nonzero. As $k T=1 /(8 \pi M)$ and $A=16 \pi M^{2}$ (in this article and earlier ones we only consider Schwarzschild black hole for simplicity), we can write

$$
\frac{1}{\tau}=N \frac{\alpha_{0}}{A_{B H}^{3 / 2}}
$$

for some constant $\alpha_{0}$ calculable from (20) and (22). Therefore, for macroscopic black hole, we can write

$$
\frac{x_{A_{i}, \Delta t}}{\Delta t}=N_{i} \frac{\alpha_{0}}{A_{B H}^{3 / 2}}
$$

which, when plugged in (21), is given by

$$
\frac{x_{A_{i}, \Delta t}}{\Delta t}=\frac{d_{i} e^{-A_{i} / 4}}{\sqrt{A_{B H}}}\left(\frac{\alpha_{0}}{\sum_{i} d_{i} A_{i} e^{-A_{i} / 4}}\right)
$$

Now, let us assume that this formula holds for single-partition black hole and further assume that the left-hand side of the above equation is constant, which is reasonable as $N_{i}$, being 1 , is constant. Then, as the factors in the parenthesis in the above equation are merely constant, we immediately see that (7) and (10) are reproduced.

\section{Discussions and Conclusions}

One may argue that the table is not fitted correctly as it is fitted with Bose-Einstein distribution factor $e^{A / 4}-1$ while the table should be fitted with the Maxwell-Boltzmann distribution factor $e^{A / 4}$ if author's earlier work [11] is correct. This is true, but the difference is insignificant as the lowest value for $e^{A / 4}$ is about 85 , which means that extra " -1 " would not significantly affect the fitting.

In this paper, we showed what the thermodynamics of single-partition black hole implies about the degeneracy of area spectrum up from $y=1$ to $y=15$. For your information, the number of degeneracies for $A(y)$ up to $y=65$ is available at (http://youngsubyoon.com/65.csv). If you add them up, you get $K(A)$.

In conclusion, we strongly believe that the extremely strange behaviors of Table 1 (the approximate constancy of $C$ and the relation not holding beginning from exactly $y=$ 16) are not a mere coincidence, instead they indicate the correctness of the area spectrum presented in [12].

\section{Data Availability}

The data are available at youngsubyoon.com/65.csv.

\section{Conflicts of Interest}

The authors declare that they have no conflicts of interest.

\section{References}

[1] S. W. Hawking, "Particle creation by black holes," Communications in Mathematical Physics, vol. 43, no. 3, pp. 199-220, 1975.

[2] C. Rovelli and L. Smolin, "Loop space representation of quantum general relativity," Nuclear Physics. B. Theoretical, Phenomenological, and Experimental High Energy Physics. Quantum Field Theory and Statistical Systems, vol. 331, no. 1, pp. 80152, 1990.

[3] C. Rovelli and L. Smolin, "Discreteness of area and volume in quantum gravity," Nuclear Physics B, vol. 442, no. 3, pp. 593-619, 1995.

[4] S. Frittelli, L. Lehner, and C. Rovelli, "The complete spectrum of the area from recoupling theory in loop quantum gravity," Classical and Quantum Gravity, vol. 13, no. 11, pp. 2921-2931, 1996.

[5] A. Ashtekar and J. Lewandowski, "Quantum theory of geometry. I. Area operators," Classical and Quantum Gravity, vol. 14, no. 1A, pp. A55-A81, 1997.

[6] C. Rovelli, "Black hole entropy from loop quantum gravity," Physical Review Letters, vol. 77, no. 16, pp. 3288-3291, 1996.

[7] J. D. Bekenstein and V. F. Mukhanov, "Spectroscopy of the quantum black hole," Physics Letters B, vol. 360, no. 1-2, pp. 7-12, 1995.

[8] M. Barreira, M. Carfora, and C. Rovelli, "Physics with nonperturbative quantum gravity: radiation from a quantum black hole," General Relativity and Gravitation, vol. 28, no. 11, pp. 1293-1299, 1996.

[9] K. V. Krasnov, "Quantum geometry and thermal radiation from black holes," Classical and Quantum Gravity, vol. 16, no. 2, pp. 563-578, 1999. 
[10] Y. Yoon, "Quantum corrections to the Hawking radiation spectrum," Journal of the Korean Physical Society, vol. 68, no. 6, pp. 730-734, 2016.

[11] Y. Yoon, "Maxwell-Boltzmann type Hawking radiation," Modern Physics Letters A, vol. 32, no. 12, 1750071, 4 pages, 2017.

[12] B. Kong and Y. Yoon, "Black hole entropy predictions without the Immirzi parameter and Hawking radiation of a singlepartition black hole," Journal of the Korean Physical Society, vol. 68, no. 6, pp. 735-751, 2016.

[13] J. D. Bekenstein, "Black holes and entropy", Physical Review D: Particles, Fields, Gravitation and Cosmology, vol. 7, pp. 23332346, 1973.

[14] T. Tanaka and T. Tamaki, "Black hole entropy for the general area spectrum," Journal of Physics: Conference Series, vol. 229, p. 012080, 2010.

[15] D. J. Griffiths, Introduction to quantum mechanics, Prentice Hall, Upper Saddle River, NJ, 2005. 

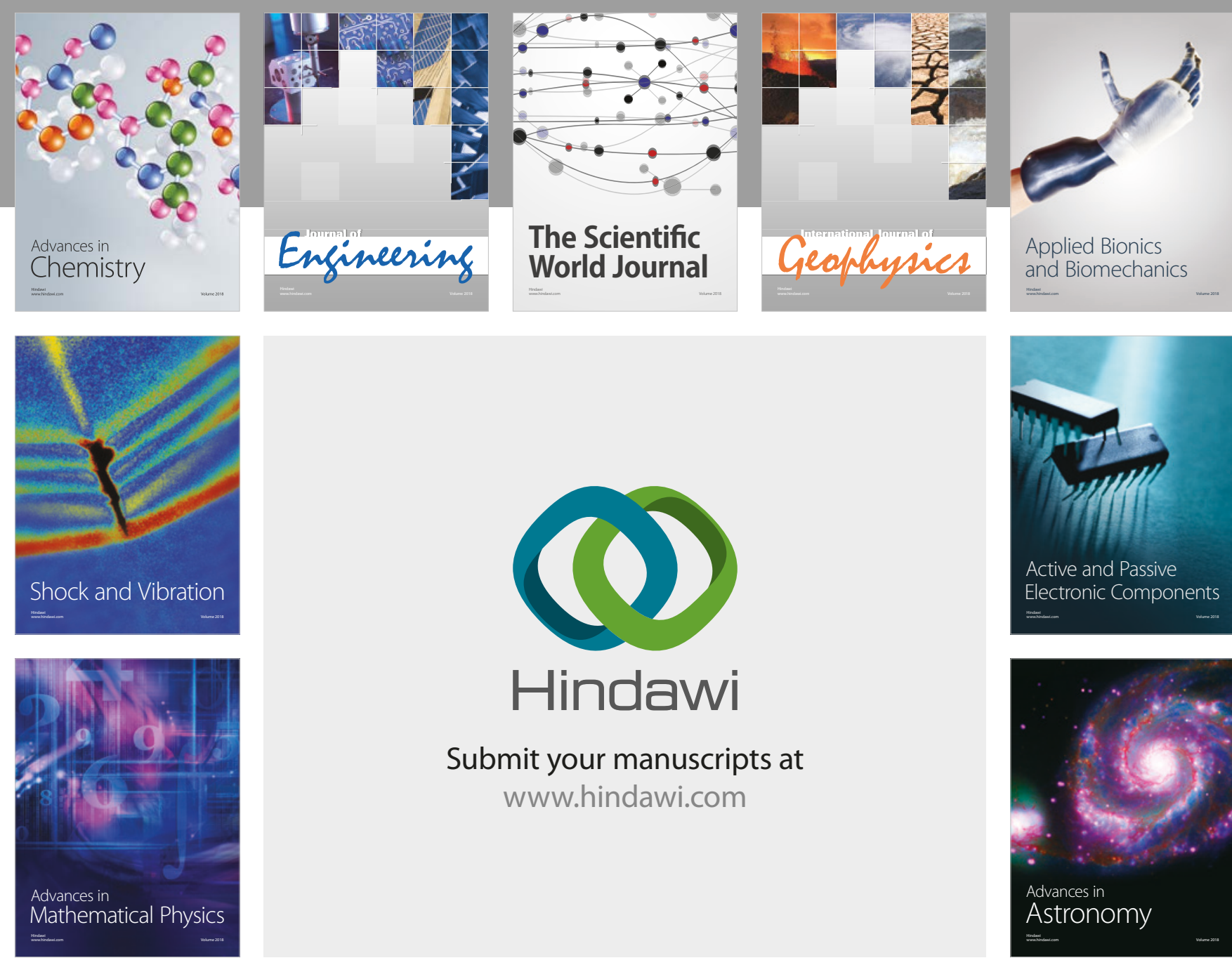

Submit your manuscripts at

www.hindawi.com



\section{Rotcting Machinery}
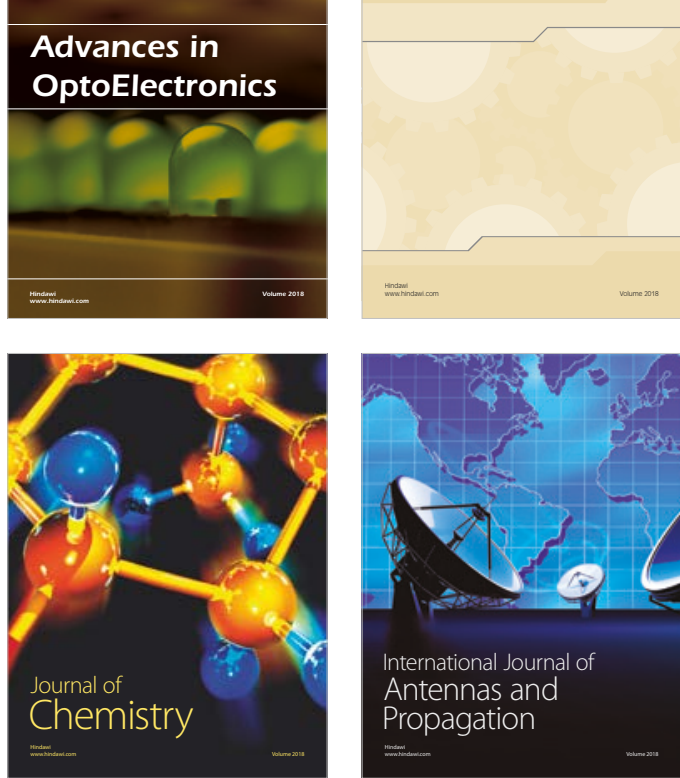

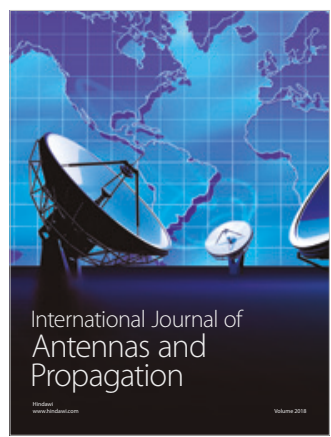

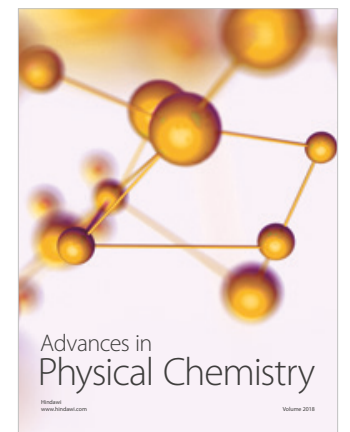

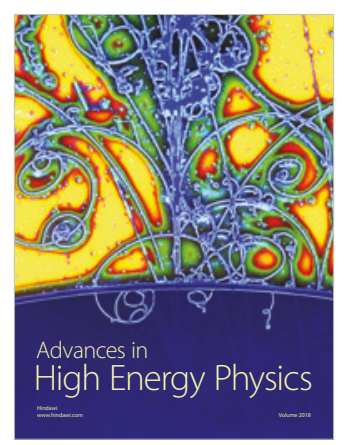

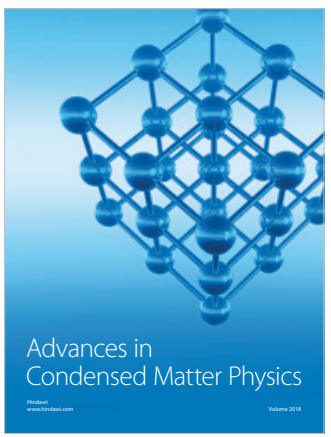

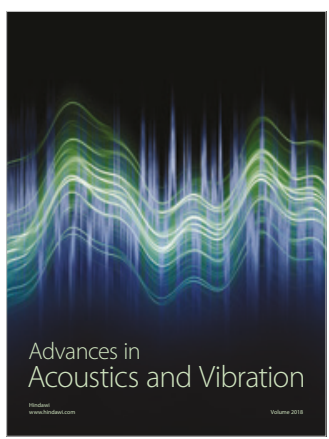

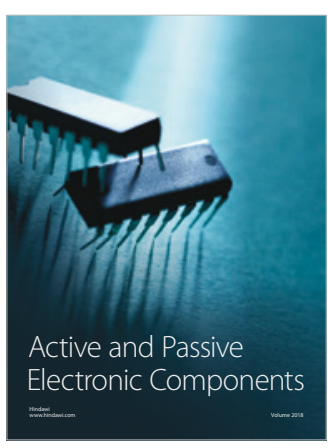
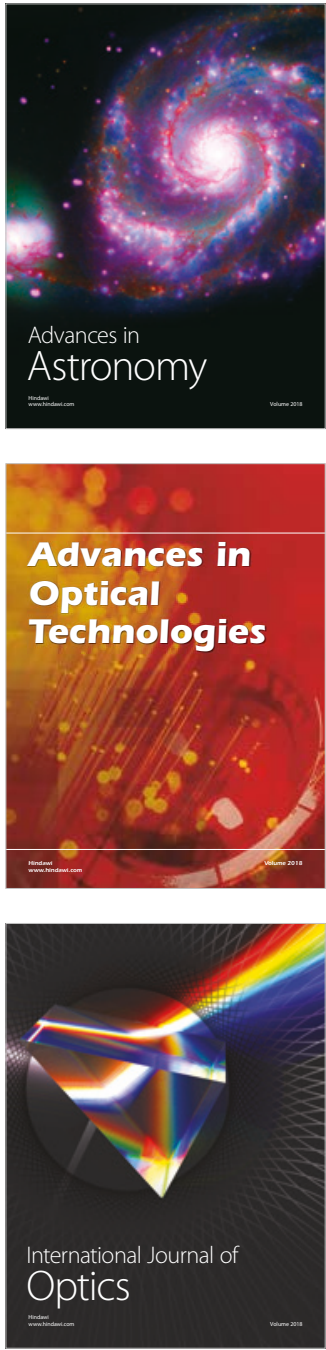